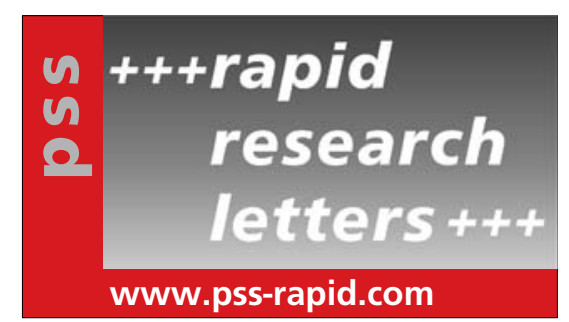

\title{
Silicon nanoparticle charge trapping memory cell
}

\author{
Nazek El-Atab", ${ }^{*, 1}$ Ayse Ozcan ${ }^{2,3}$, Sabri Alkis, ${ }^{2,3}$, Ali K. Okyay ${ }^{2,3,4}$, and Ammar Nayfeh ${ }^{1}$ \\ ${ }^{1}$ Institute Center for Microsystems - iMicro, Department of Electrical Engineering and Computer Science (EECS), \\ Masdar Institute of Science and Technology Abu Dhabi, United Arab Emirates \\ ${ }^{2}$ UNAM-National Nanotechnology Research Center, Bilkent University, 06800 Ankara, Turkey \\ ${ }^{3}$ Institute of Materials Science and Nanotechnology, Bilkent University, 06800 Ankara, Turkey \\ ${ }^{4}$ Department of Electrical and Electronics Engineering, Bilkent University, 06800 Ankara, Turkey
}

Received 3 April 2014, revised 7 May 2014, accepted 7 May 2014

Published online 12 May 2014

Keywords Si nanoparticles, $\mathrm{ZnO}$, charge trapping memories, $\mathrm{Al}_{2} \mathrm{O}_{3}$, phonon-assisted tunneling, atomic layer deposition

* Corresponding author: e-mail nelatab@masdar.ac.ae

A charge trapping memory with $2 \mathrm{~nm}$ silicon nanoparticles (Si NPs) is demonstrated. A zinc oxide ( $\mathrm{ZnO})$ active layer is deposited by atomic layer deposition (ALD), preceded by $\mathrm{Al}_{2} \mathrm{O}_{3}$ which acts as the gate, blocking and tunneling oxide. Spin coating technique is used to deposit Si NPs across the sample between $\mathrm{Al}_{2} \mathrm{O}_{3}$ steps. The $\mathrm{Si}$ nanoparticle memory exhibits a threshold voltage $\left(V_{\mathrm{t}}\right)$ shift of $2.9 \mathrm{~V}$ at a negative programming voltage of $-10 \mathrm{~V}$ indicating that holes are emitted from channel to charge trapping layer. The negligible measured $V_{\mathrm{t}}$ shift without the nanoparticles and the good re- tention of charges ( $>10$ years) with Si NPs confirm that the $\mathrm{Si}$ NPs act as deep energy states within the bandgap of the $\mathrm{Al}_{2} \mathrm{O}_{3}$ layer. In order to determine the mechanism for hole emission, we study the effect of the electric field across the tunnel oxide on the magnitude and trend of the $V_{\mathrm{t}}$ shift. The $V_{\mathrm{t}}$ shift is only achieved at electric fields above $1 \mathrm{MV} / \mathrm{cm}$. This high field indicates that tunneling is the main mechanism. More specifically, phonon-assisted tunneling (PAT) dominates at electric fields between $1.2 \mathrm{MV} / \mathrm{cm}<E<2.1 \mathrm{MV} / \mathrm{cm}$, while FowlerNordheim tunneling leads at higher fields $(E>2.1 \mathrm{MV} / \mathrm{cm})$.
1 Introduction Silicon nanoparticles (NPs) are considered as good candidates for charge trapping and storage elements for future low voltage and high density nonvolatile charge trapping memory devices [1-4]. In addition, it is validated recently that the use of $\mathrm{ZnO}$ leads through promising low-cost memory device fabrications [5-7]. Earlier, we demonstrated a $\mathrm{ZnO}$-based memory with $2 \mathrm{~nm} \mathrm{Si}$ NPs embedded in a $\mathrm{ZnO}$ charge trapping layer [4]. The structure showed a large memory window at very low operating voltages. This was attributed to the Poole-Frenkel emission of charges at low electric fields. In this work, a memory structure with only $2 \mathrm{~nm}$ average size Si NPs and without $\mathrm{ZnO}$ charge trapping layer is investigated. Compared to Ref. [4], this structure enables a better understanding of the charge trapping characteristics of the $2 \mathrm{~nm} \mathrm{Si}$ NPs. Also, the elimination of the $\mathrm{ZnO}$ layers reduces the gate stack thickness which would allow for further increasing the density of the memories. A diagram regarding the fabricated memory device is given along with a transmis- sion electron microscope (TEM) image of Si NPs and the effect of the Si NPs on the device performance is shown with electrical measurements. The emission mechanism is determined by studying the $V_{\mathrm{t}}$ shift vs. electric field across the tunnel oxide.

2 Fabrication Dispersed silicon nanoparticles of size $5-100 \mathrm{~nm}$ are first produced by applying a pulsed laser on a silicon target wafer which is submerged in deionized water. The femtosecond pulsed laser has a pulse duration of $200 \mathrm{fs}$, a wavelength $\lambda=800 \mathrm{~nm}$, an average output power of $1.6 \mathrm{~W}$, and a pulse repetition rate of $1 \mathrm{kHz}$ which corresponds to an energy of $1.6 \mathrm{~mJ}$. The Si NPs are then treated in an ultrasound bath for $200 \mathrm{~min}$ with an operating frequency of $40 \mathrm{kHz}$. Finally, filters with pore size of $100 \mathrm{~nm}$ are used to remove large particles leaving a solution of Si NPs of predominately $2 \mathrm{~nm}$ in size (ranging from $1-5.5 \mathrm{~nm}$ ) [8]. A TEM image of the synthesized Si NPs is depicted in Fig. 1a. The image 

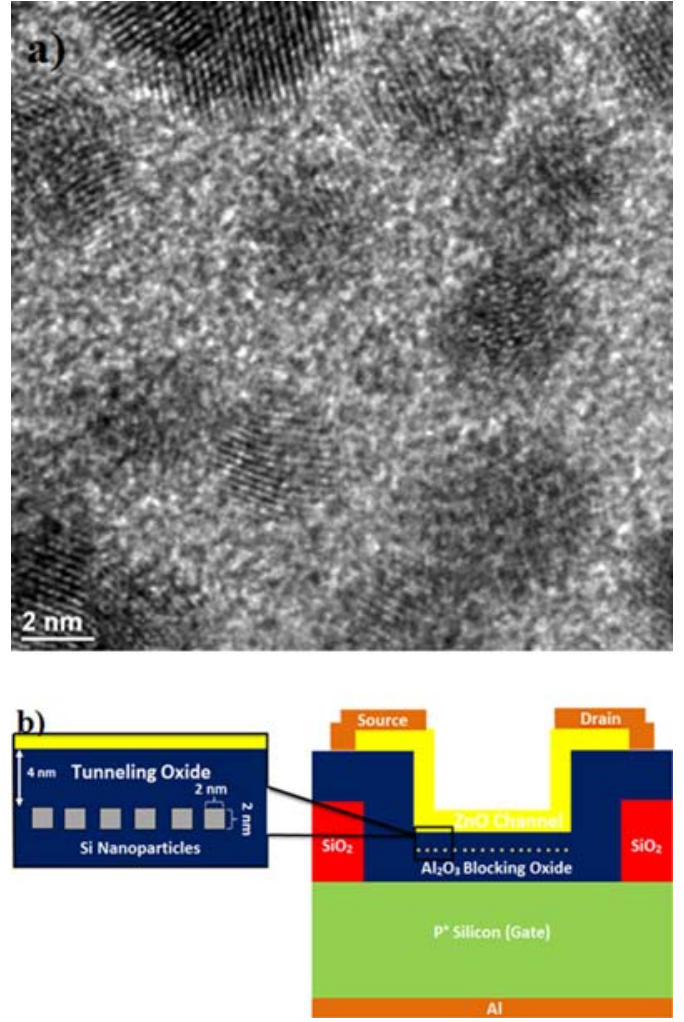

Figure 1 (a) TEM image of the synthesized silicon nanoparticles. (b) Cross-sectional illustration of the fabricated charge trapping memory with $2 \mathrm{~nm}$ Si nanoparticles.

shows that ultra-small and dispersed Si NPs are fabricated.

The bottom-gate memory cells are fabricated on a highly doped $(10-18 \mathrm{~m} \Omega \mathrm{cm})$ p-type (111) Si wafer. First, a $360 \mathrm{~nm}$ thick $\mathrm{SiO}_{2}$ layer for device isolation is deposited using plasma enhanced chemical vapor deposition (PECVD). Then, the $\mathrm{SiO}_{2}$ layer is patterned by optical lithography and etched using the buffered oxide etch (BOE) for $7 \mathrm{~min}$. Using a Cambridge Nanotech Savannah 100 atomic layer deposition (ALD) system, a $15 \mathrm{~nm}$ thick $\mathrm{Al}_{2} \mathrm{O}_{3}$ blocking oxide is deposited at $250{ }^{\circ} \mathrm{C}$.

Next, Si NPs are delivered by spin coating the NPs solution at a speed of $700 \mathrm{rpm}$ and an acceleration of $250 \mathrm{rpm} / \mathrm{sec}$ for $10 \mathrm{sec}$. A $4 \mathrm{~nm}$ thick $\mathrm{Al}_{2} \mathrm{O}_{3}$ tunneling oxide and an $11 \mathrm{~nm}$ thick $\mathrm{ZnO}$ channel are then deposited by ALD. After patterning the $\mathrm{ZnO}$ channel by optical lithography, it is etched in a solution of $98: 2 \mathrm{H}_{2} \mathrm{O}: \mathrm{H}_{2} \mathrm{SO}_{4}$. The source and drain contacts are created by thermally evaporating $100 \mathrm{~nm} \mathrm{Al}$ followed by lift off. Lastly, rapid thermal annealing (RTA) in forming gas $\left(\mathrm{H}_{2}: \mathrm{N}_{2} 5: 95\right)$ at $400{ }^{\circ} \mathrm{C}$ for $10 \mathrm{~min}$ is performed on the devices. The fabricated memory cell structure is depicted in Fig. 1b.

3 Experimental characterization The charging effect of the Si NPs is analyzed by studying the $I_{\text {drain }}-V_{\text {gate }}$ curves of the programmed and erased states of memory devices with and without Si NPs. Using the Agilent-
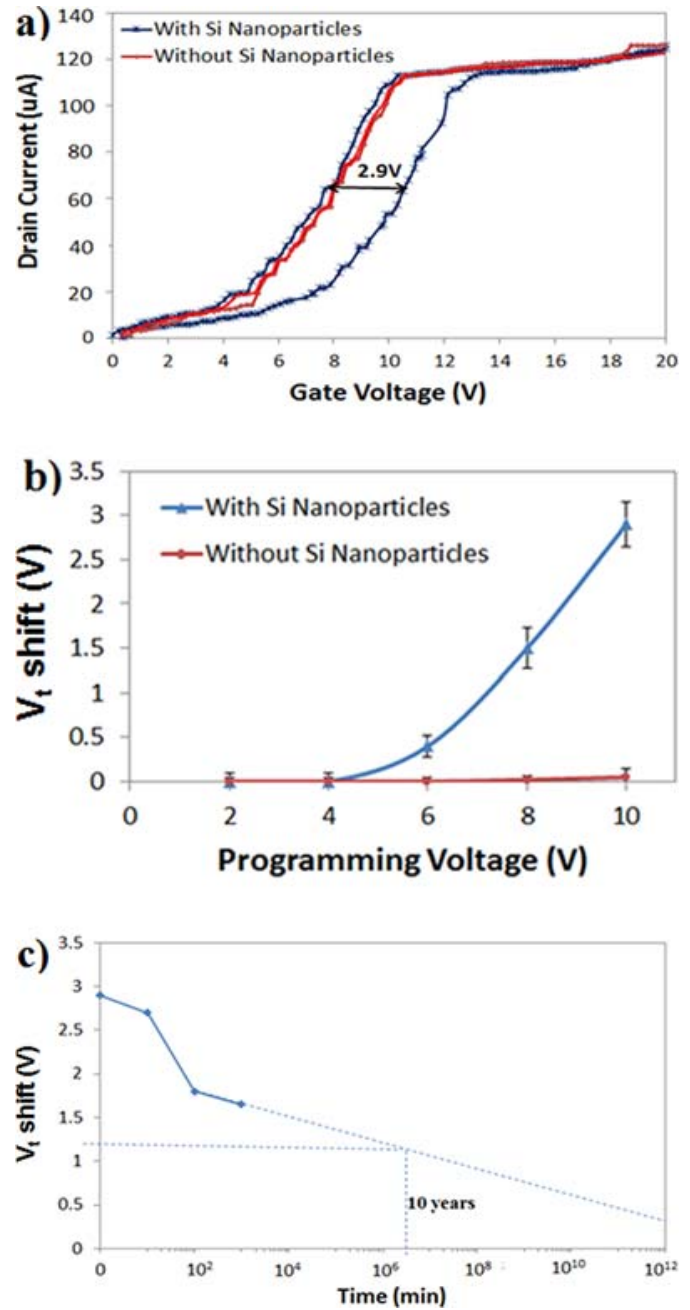

Figure 2 (a) $I_{\mathrm{d}}-V_{\mathrm{g}}$ of the memory showing $V_{\mathrm{t}}$ shift with and without $\mathrm{Si}$ nanoparticles. The memory is programmed by applying $V_{\mathrm{g}}=-10 \mathrm{~V}$ for $5 \mathrm{sec}$. (b) Threshold voltage shift vs. programming voltage with and without $\mathrm{Si}$ NPs. (c) $V_{\mathrm{t}}$ shift vs. time extrapolated to 10 years with $2 \mathrm{~nm}$ Si nanoparticles.

Signatone B1505A device analyzer, the memory cells are programmed/erased by applying a gate voltage of $-10 \mathrm{~V} / 10 \mathrm{~V}$ for $5 \mathrm{sec}$. Then, the gate voltage is swept from $0 \mathrm{~V}$ up to $20 \mathrm{~V}$ with a drain voltage $V_{\mathrm{d}}$ of $10 \mathrm{~V}$ in order to read the state of the cell. In fact, applying a negative gate voltage would program the memory and a positive gate voltage would erase it. This confirms that holes are emitted into the Si NPs. The obtained $V_{\mathrm{t}}$ shift with Si NPs is $2.9 \mathrm{~V}$ while it is negligible without NPs as shown in Fig. 2a. This proves that $\mathrm{Al}_{2} \mathrm{O}_{3}$ of thickness up to $19 \mathrm{~nm}$ has a negligible trapping density which makes this oxide an excellent candidate for tunnel and blocking oxides. Assuming the shift in the threshold voltage is mainly due to the trapped charges in the trapping layer, the charge trap states density can be calculated by adopting Eq. (1) [9],

$$
Q=\frac{C_{\mathrm{t}} \cdot \Delta V_{\mathrm{t}}}{2 \cdot q},
$$


where $C_{\mathrm{t}}$ is the capacitance of the charge trapping layer per unit area, $\Delta V_{\mathrm{t}}$ is the $V_{\mathrm{t}}$ shift, and $q$ is the elementary charge. For a $2.9 \mathrm{~V} V_{\mathrm{t}}$ shift, the charge trap states density is roughly $7 \times 10^{12} \mathrm{~cm}^{-2}$.

In addition, the erased state (also fresh state) of the memory with Si NPs is shifted to the right with respect to the eased state of the memory without NPs as shown in Fig. 2a. The right shift of the threshold voltage indicates that the $2 \mathrm{~nm}$ laser synthesized Si NPs are initially negatively charged. The value of the initial negative charge on the Si NPs can be calculated using Eq. (1) which results in a charge density of $7 \times 10^{12} \mathrm{~cm}^{-2}$. During the programming operation, at a negative applied gate voltage, the Si NPs initial negative charge increases the electric filed across the tunnel oxide, thus enhancing the effect of hole injection.

Additionally, the mean and standard deviation of the measured $V_{\mathrm{t}}$ shifts at different programming voltages with and without nanoparticles are shown in Fig. 2b. With Si NPs, the $V_{\mathrm{t}}$ shift at a programming voltage of $-8 \mathrm{~V}$ is around $1.4 \mathrm{~V}$. Figure $2 \mathrm{~b}$ confirms that Si NPs act as charge trapping centers with high trapping density. Also, the standard deviation of the measured $V_{\mathrm{t}}$ shifts with Si NPs are higher owing to the non-uniformity of the distribution of the Si NPs by spin coating in addition to the different number and size of the Si NPs creating the charge trapping layer of each memory cell.

Moreover, the retention characteristic of the Si NPs is analyzed by plotting the $V_{\mathrm{t}}$ shift vs. time as shown in Fig. 2c. The curve is extrapolated to 10 years where the memory cell exhibits a noticeable $V_{\mathrm{t}}$ shift of $1.25 \mathrm{~V}$, which means a loss of $57 \%$ of the initial charge in 10 years. The retention of the memory cell is due to the good confinement of holes in the nanoparticles.

4 Analysis In order to determine the mechanism of holes emission, $V_{\mathrm{t}}$ shift versus the square of the electric field across the tunnel oxide is plotted in Fig. 3a. The electric field is calculated using Synopsys Sentaurus Physics based TCAD simulations. The details of the TCAD model can be found in Refs. [10,11]. In the simulations, size quantization effects are included by using the density gradient model. Also, the Lucent mobility model is used and high-field saturation effects are accounted for. The $V_{\mathrm{t}}$ is extracted using the maximum transconductance method. The linear relation depicted in Fig. 3a between $V_{\mathrm{t}}$ shift and the square of the electric field confirms that phonon-assisted tunneling is the main mechanism for hole emission from the channel to the Si NPs charge trapping layer at electric fields higher than $1.2 \mathrm{MV} / \mathrm{cm}$.

The emission rate in PAT increases exponentially with the square of the electric field intensity according to Eq. (2) $[12,13]$ :

$$
\frac{e(E)}{e(0)}=\mathrm{e}^{\frac{E^{2}}{E_{\mathrm{c}}^{2}}}
$$
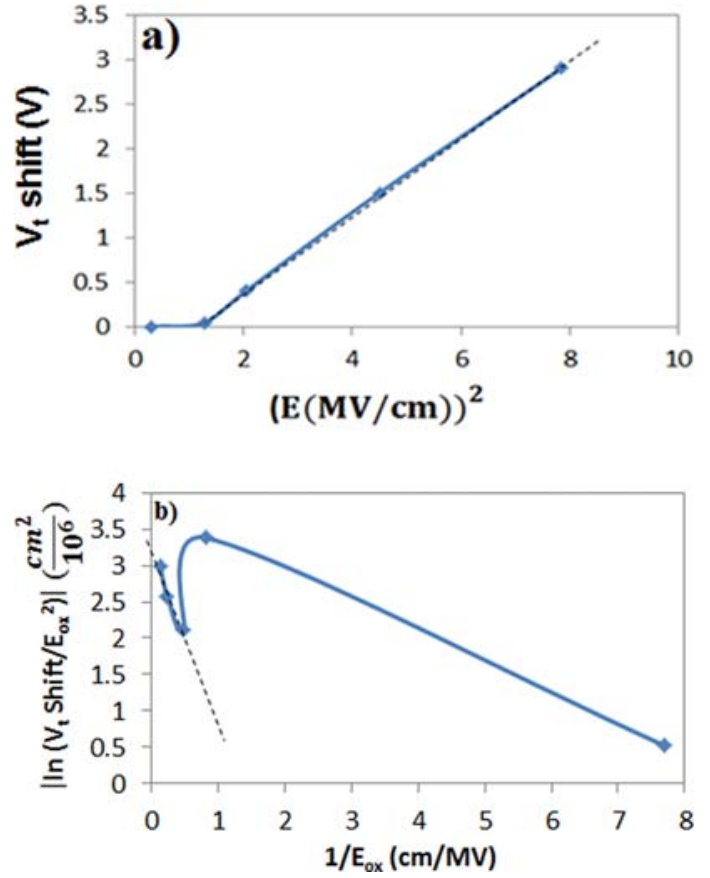

Figure 3 (a) $V_{\mathrm{t}}$ shift vs. square of the electric field across the tunnel oxide showing a linear dependence indicating phononassisted tunneling for emission. (b) The natural logarithm of the $V_{\mathrm{t}}$ shift over the square of the electric field is plotted vs. the reciprocal of the electric field. The linear trend indicates that Fowler-Nordheim $(\mathrm{F}-\mathrm{N})$ is the dominant emission mechanism at an oxide electric field of $2.1 \mathrm{MV} / \mathrm{cm}$.

where $e(E)$ and $e(0)$ are the thermal ionization probabilities with and without electric field $E$, respectively, and $E_{\mathrm{c}}$ is the characteristic field intensity. PAT is an inelastic tunneling mechanism where the holes in the channel gain enough energy from phonons and tunnel through the tunnel oxide to the Si NPs. Another possible mechanism in PAT is that holes in the channel tunnel to a trap within the tunnel oxide, lose some energy by emitting a phonon, and tunnel again to the charge trapping layer. However, since the memory without NPs showed negligible $V_{\mathrm{t}}$ shift, then the traps in the $\mathrm{Al}_{2} \mathrm{O}_{3}$ are expected to be near the interface between $\mathrm{Al}_{2} \mathrm{O}_{3}$ and the $\mathrm{ZnO}$ channel so that they do not trap charges with a long retention time.

In addition, the linear trend shown in Fig. 3b, where the natural logarithm of the $V_{\mathrm{t}}$ shift over the square of the electric field across the tunnel oxide is plotted versus the inverse of the field, shows that at electric fields larger than $2.1 \mathrm{MV} / \mathrm{cm}, \mathrm{F}-\mathrm{N}$ tunneling is valid. However, since $\mathrm{F}-\mathrm{N}$ tunneling has a stronger dependence than PAT on the electric field, then the dominant mechanism at higher fields $(E>2.1 \mathrm{MV} / \mathrm{cm})$ is $\mathrm{F}-\mathrm{N}$ tunneling. $\mathrm{F}-\mathrm{N}$ tunneling is considered the tunneling mechanism which requires the highest electric field across the tunel oxide to be applicable. Therefore, a triangular energy barrier is formed due to the high electric field, and holes are injected by tunneling into the valence band of the tunnel oxide through the formed triangular barrier and then are swept by the electric field 


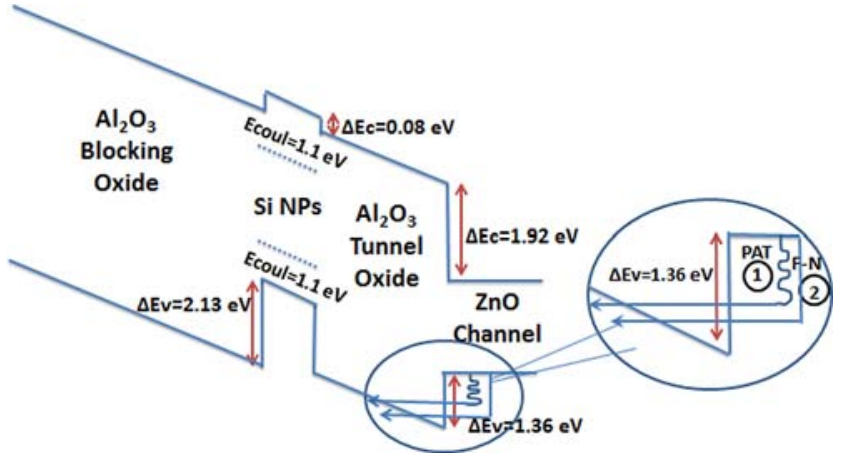

Figure 4 Energy band diagram of the Si NPs charge trapping memory at a negative applied bias. (1) Holes gain a thermal energy and tunnel through the tunnel oxide through phonon-assisted tunneling (PAT), (2) holes tunnel through a triangular energy barrier by Fowler-Nordheim $(\mathrm{F}-\mathrm{N})$.

into the Si NPs. The emission rate of charges in $\mathrm{F}-\mathrm{N}$ tunneling follows Eq. (3) [14]:

$$
J=C_{1} E_{\mathrm{ox}}^{2} \mathrm{e}^{\frac{C_{2}}{E_{\mathrm{ox}}}},
$$

where $J$ is the $\mathrm{F}-\mathrm{N}$ tunneling current, $E_{\mathrm{ox}}$ is the electric field across the tunnel oxide, and $C_{1}$ and $C_{2}$ are constants in terms of the effective mass and barrier height. Furthermore, the negatively charged Si NPs enhance the electric field across the tunnel oxide allowing for PAT and $\mathrm{F}-\mathrm{N}$ mechanisms to be applicable at lower electric fields.

Using the material properties of $\mathrm{ZnO}, \mathrm{Al}_{2} \mathrm{O}_{3}[7,15$, 16], and $2 \mathrm{~nm}$ Si nanoparticles [17-19], the energy band diagram of the memory cell with Si NPs is constructed and shown in Fig. 4. The changes in the electronic structure of the Si NPs due to quantum confinement in 0-D [17] and to the increased charging energy are taken into account when constructing the energy band diagram. The Coulomb charging energy which represents the energy needed to add a single charge to the nanoparticle is given by Eq. (4),

$$
E=\frac{q^{2}}{C}
$$

where $q$ is the Coulomb charge and $C$ is the capacitance of the nanoparticle given by $4 \pi \varepsilon R$, where $\varepsilon$ is the dielectric constant and $R$ is the radius of the spherical nanoparticle.

The Coulomb charging energy of Si NPs of size $2 \mathrm{~nm}$ is calculated to be $1.1 \mathrm{eV}$ and is shown in the energy band diagram plot. At $2 \mathrm{~nm}$ size scale, the "effective mass" for holes and electrons is similar, thus the increase in energy gap of the Si NPs from bulk silicon is partitioned and added equally to both the valence band and conduction band [20]. Figure 4 shows that the conduction band offset between channel and tunnel oxide $\left(\Delta E_{\mathrm{c}}=1.92 \mathrm{eV}\right)$ is larger than the valence band offset $\left(\Delta E_{\mathrm{v}}=1.36 \mathrm{eV}\right)$, which makes the tunneling probability of holes through the tunnel oxide much higher than electrons probability of tunneling.
Additionally, because the electron affinity is reduced as the size of the NPs shrinks [19], the conduction band minimum of the Si NPs is above that of the adjacent $\mathrm{Al}_{2} \mathrm{O}_{3}$ by $0.08 \mathrm{eV}$ which might prevent electrons storage, but the valence band minimum of the Si NPs is beyond that of the adjacent $\mathrm{Al}_{2} \mathrm{O}_{3}$ by $2.13 \mathrm{eV}$ so a quantum well is formed where holes can be confined in. This analysis supports the observed hole trapping in the Si NPs. Figure 4 illustrates the PAT and $\mathrm{F}-\mathrm{N}$ mechanisms for hole emission from the channel to Si NPs. In the earlier work with the $\mathrm{ZnO}$ layer, lower operating voltage was achieved since the mechanism driving the emission was Poole-Frenkel effect (PFE). The electric field needed is less than $1 \mathrm{MV} / \mathrm{cm}$ since the $\mathrm{ZnO}$ layer adds available trap states to assist the emission [4].

5 Conclusion A $\mathrm{Si}$ nanoparticle charge trapping memory is demonstrated. A $2.9 \mathrm{~V} V_{\mathrm{t}}$ shift at $-10 \mathrm{~V}$ programming voltage is achieved with the $\mathrm{Si}$ NPs. This confirms that the laser-synthesized Si NPs behave as trapping centers with high trapping density. The dominant mechanism for hole emission is found to be $\mathrm{PAT}$ when $1.2 \mathrm{MV} / \mathrm{cm}<E<2.1 \mathrm{MV} / \mathrm{cm}$, and $\mathrm{F}-\mathrm{N}$ when $E>2.1 \mathrm{MV} / \mathrm{cm}$. The negatively charged nature of the $\mathrm{Si}$ NPs helps in increasing the electric filed across the tunnel oxide during the program function, allowing for an enhanced hole injection through PAT and F-N. The results highlight the importance of the emission mechanism on the magnitude of the voltage needed to achieve the memory effect. The good retention time of the memory allows for further scaling of the tunnel oxide without compromising the $V_{\mathrm{t}}$ shift or retention. Finally, these results show that Si NPs are a good candidate for charge trapping layers in future low-cost nonvolatile memory devices.

Acknowledgements We gratefully acknowledge financial support for this work provided by the Advanced Technology Investment Company (ATIC) Grant 12RAZB7. This work was supported in part by TUBITAK Grants 109E044, 112M004, $112 \mathrm{E} 052$ and $113 \mathrm{M} 815$.

Supporting Information Available online at www.pssrapid.com.

\section{References}

[1] N. Nedev, D. Nesheva, E. Manolov, R. Brüggemann, S. Meier, Z. Levi, and R. Zlatev, J. Phys. Conf. Ser. 113, 012034-012039 (2008).

[2] L. Wang, M. Ji, S. Zhu, H. Tu, and J. Du, J. Phys. Conf. Ser. 152, 012028-012033 (2009).

[3] H. H. I. Hanafi, S. Tiwari, and I. Khan, IEEE Trans. Electron Devices 43, 1553-1558 (1996).

[4] N. El-Atab, A. Ozcan, S. Alkis, A. K. Okyay, and A. Nayfeh, Appl. Phys. Lett. 104, $013112-013115$ (2014).

[5] N. El-Atab, S. Alqatari, F. B. Oruc, T. Souier, M. Chiesa, A. K. Okyay, and A. Nayfeh, AIP Adv. 3, 102119-102126 (2013). 
[6] F. B. Oruc, F. Cimen, A. Rizk, M. Ghaffari, A. Nayfeh, and A. K. Okyay, IEEE Electron Device Lett. 33, 1714-1716 (2012).

[7] N. El-Atab, A. Rizk, A. K. Okyay, and A. Nayfeh, AIP Adv. 3, 112116 (2013).

[8] S. Alkis, A. K. Okyay, and B. Ortac, J. Phys. Chem. 116, 3432-3436 (2012).

[9] C. W. Liu, C. L. Cheng, S. W. Huang, J. T. Jeng, S. H. Shiau, and B. T. Dai, Appl. Phys. Lett. 91, 042107 (2007).

[10] A. Alnuaimi, A. Nayfeh, and V. Koldyaev, J. Appl. Phys. 113, 044513-044519 (2013)

[11] J. Bu and M. H. White, Solid-State Electron. 45, 113-120 (2001).

[12] S. D. Ganichev, W. Prettl, and P. G. Huggard, Phys. Rev. 71, 3882-3885 (1993)

[13] S. D. Ganichev, I. N. Yassievich, W. Prettl, J. Diener, B. K. Meyer, and K. W. Benz, Phys. Rev. Lett. 75, 1590-1594 (1995).
[14] S. M. Sze, Nonvolatile Memory Devices, in: Physics of Semiconductor Devices, 3rd ed. (Wiley, 2007, New Jersey), chap. 6, s. 7, p. 353 .

[15] M. L. Huang, Y. C. Chang, C. H. Chang, T. D. Lin, J. Kwo, T. B. Wu, and M. Hong, Appl. Phys. Lett. 89, 012903012906 (2006).

[16] G. D. Wilk, R. M. Wallace, and J. M. Anthony, J. Appl. Phys. 89, 5243 (2001).

[17] J. P. Proot, C. Delerue, and G. Allan, Appl. Phys. Lett. 61, 1948-1950 (1992).

[18] C. Delerue and G. Allan, Appl. Phys. Lett. 88, 173117173120 (2006).

[19] D. V. Melnikov and J. R. Chelikowsky, Phys. Rev. B 69, 113305-113309 (2004).

[20] O. M. Nayfeh, Ph.D. dissertation, EECS, MIT, Cambridge, MA (2009). 